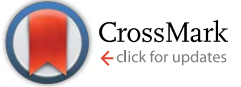

Cite this: RSC Adv., 2017, 7, 11969

Received 31st December 2016 Accepted 2nd February 2017

DOI: $10.1039 / \mathrm{c} 6 \mathrm{ra} 28875 \mathrm{~h}$

rsc.li/rsc-advances

\section{Fabrication of arrayed triangular micro-cavities for SERS substrates using the force modulated indention process}

\begin{abstract}
Yongda Yan, ${ }^{\star a b}$ Jingran Zhang, ${ }^{\text {ab }}$ Ping $\mathrm{Xu}^{\mathrm{c}}$ and Peng Miao
Based on the tip-based continuous indentation process, a novel method for the fabrication of periodic arrayed triangular micro-cavities on copper (Cu) surfaces is presented. The indentation force is modulated and the indentation speed and moving velocity of the precision stage used to drive the workpiece are optimized to improve the machining efficiency. The deformation property of the pile-ups at the side of the pyramidal cavity is studied. Due to the overlap of the pile-ups of adjacent micro pyramidal cavities, two and three dimensional arrayed nanostructures are successfully achieved. Then, the structured $\mathrm{Cu}$ (110) surface is used as a surface-enhanced Raman scattering (SERS) substrate with the rhodamine 6G (R6G) probe molecule as the detecting target in the present study. Experimental results show that the Raman intensity is enhanced with a decrease in the moving velocity of the precision stage. SERS enhancement factors within the range of $10^{5}$ to $3 \times 10^{6}$ are achieved on the structured $\mathrm{Cu}(110)$ surface, which demonstrates that this method is reliable, replicable, homogeneous and inexpensive for the fabrication of SERS substrates.
\end{abstract}

\section{Introduction}

Micro and nano machining technology has become a important research topic and is attracting increasing research attention. ${ }^{\mathbf{1 - 3}}$ Various micro/nanostructures, including micro/nanowells, micro/nanochannels, micro/nanodomes and even three dimensional microstructures, which play important roles in microchip cooling, ${ }^{4,5}$ solar cells, ${ }^{6,7}$ plasmonic-enhanced photovoltaics $^{8-10}$ and surface-enhanced Raman scattering (SERS), ${ }^{11,12}$ have been successfully fabricated via the existing micro/ nanomachining methods.

Metal micro/nanostructures have been already verified for use as SERS substrates. Several years ago, researchers found that the rough surface of some noble metals (gold, silver and copper), nanoparticles or specific arrayed structures could enhance energy transfer between a metal substrate and molecules and avoid fluorescence background interference. Compared with traditional Raman spectroscopy, surface enhanced Raman spectroscopy provides improved detection sensitivity. Therefore, SERS applications have experienced a huge growth in the past decade and span a wide range of

\footnotetext{
${ }^{a}$ The State Key Laboratory of Robotics and Systems, Robotics Institute, Harbin Institute of Technology, Harbin 150080, P. R. China.E-mail: yanyongda@hit.edu.cn; Fax: +86451-86415244; Tel: +86-451-86412924

${ }^{b}$ Center for Precision Engineering, Harbin Institute of Technology, Harbin, Heilongjiang 150001, P. R. China

${ }^{c}$ School of Chemistry and Chemical Engineering, Harbin Institute of Technology, Harbin, Heilongjiang 150001, P. R. China
}

fields, including biosensing and bioanalysis, ${ }^{\mathbf{1 3 , 1 4}}$ bacteria and virus identification, ${ }^{15,16}$ and the detection of explosives ${ }^{17,18}$ and toxic pesticides. ${ }^{19}$

Typically, micro/nanostructures are mainly machined by lithography-based technologies, including electron-beam lithography (EBL), ${ }^{20-22}$ colloidal lithography (CL), ${ }^{23,24}$ templateassisted lithography (TAL), ${ }^{25}$ nanoimprint lithography (NIL), ${ }^{26,27}$ soft interference lithography (SIL), ${ }^{28-30}$ nanosphere lithography (NSL) ${ }^{31,32}$ and laser interference lithography (LIL). ${ }^{33}$ In addition, micro/nanostructures are also machined by hybrid lithography methods combined with other methods, such as dry etching ${ }^{34-36}$ and wetting etching. ${ }^{37-41}$ For example, Ye et al. ${ }^{35}$ designed and fabricated $\mathrm{Ag}$ nanowell crystals by colloidal lithography and $\mathrm{O}_{2}$ plasma etching. The nanowells, which were $483 \mathrm{~nm}$ in width and $40 \mathrm{~nm}$ in depth, were used as a label-free biosensor. Klarite SERS substrates with the specific apex angle of $70.5^{\circ}$ on the $\mathrm{Si}(100)$ plane were fabricated using optical lithography and wetting etching method. ${ }^{39-41}$ Nanohole and nanopyramid arrayed structures were fabricated by Odom ${ }^{29}$ using the nanofabrication techniques involved in soft interference lithography (PDMS photomask), lift-off, electron-beam deposition and wetting etching. Different shapes of micro/ nanostructures with a wide range can be machined by lithography-based methods. However, the major limitations of these methods are the sophistication (complexity) of the fabrication processes, inevitable defects and high cost.

Recently, the nanoindentation method was employed for the fabrication of micro/nanostructures with ordered shapes and ordered dimension structures. Gong et al. ${ }^{42}$ machined micro/ 
nanostructures on silica film and epoxy substrates, including split rings, cavities and channels. SERS-active substrates were obtained using the transfer technique and the metal film deposition method. A self-assembled monolayer was formed on the pyramidal structures of the silver surface, and the enhancement factor of the Raman signal was $10^{7}$ to $10^{8}$ at the tip of the pyramidal structure. Chang et al. ${ }^{\mathbf{4 3}-45}$ used this method to fabricate arrayed nanocavities with a controllable indentation depth and tip-to-tip displacements on the Au surface. In addition, EMCV, adenovirus and influenza virus were detected and DNA hybridization was analyzed using the nanocavities as SERS substrates. In their studies, they used a commercial nanoindenter, which is widely used to detect the mechanical properties of hardness, elastic modulus and friction coefficient. However, the commercial nanoindentation system has the shortcomings of small machining scale, low machining speed and high cost. In addition, it is difficult to obtain more complex micro/nanostructures with a wide range using this system.

Therefore, in the present study, a novel tip-based force modulated indention method is employed to fabricate arrayed triangular micro-cavities. By controlling the period of the force signal and the machining velocity of the precision stage, the property of the formed pile-ups on the $\mathrm{Cu}(110)$ plane is studied and different arrayed micro/nano-cavities based structures are formed by the overlap of the pile-ups. Finally, the Raman intensities of R6G are detected on the arrayed structures, which are used as SERS substrates.

\section{Experimental}

\subsection{The principle of the force modulated indention process}

Fig. 1 shows a schematic of the home-built tip-based force modulated indentation system and the machining process for the fabrication of substrates with periodic micro/nano-cavities. As shown in Fig. 1(a), the $x-y$ precision stage (P-517, PI, Germany) with the movement range of $100 \mu \mathrm{m}$ is used to drive the sample to control the $x-y$ scale of the periodic micro/nanocavities array, as shown in Fig. 1(b) and (c). The $z$ axis precision stage with the range of $30 \mu \mathrm{m}$ is used to make the diamond tip with the tip radius of less than $200 \mathrm{~nm}$ (Synton-MDP, Switzerland) to penetrate the sample surface according to the normal force signal, as shown in Fig. 1(b). The strain gauge force sensor with a $50 \mathrm{~g}$ capacity (LSB-200, Futek, USA) is employed to measure the normal force signal and keeps the diamond tip contacting the sample with the required force. The three stages are all driven by a UMAC controller (Delta Tau Data Systems, USA). The force signal is also captured by a port of the same UMAC. The single crystal copper $(\mathrm{Cu}(110))$ of $10 \mathrm{~mm} \times 5$ $\mathrm{mm} \times 1 \mathrm{~mm}$ is used as the sample (Heifei Ke Jing Materials Technology Co., LTD, China). Fig. 1(d) shows a scanning electron microscopy (Helios Nanolab 600i, Germany) image of the cube corner tip with its pyramidal shape.

Based on this system, the principle of fabricating periodic micro/nano cavities with the normal force modulation is presented as follows:

(1) The $z$ axis precision stage brings the diamond tip to penetrate the sample surface to perform an indentation process. Only one cavity is obtained using this process, which is similar to the conventional indentation process.

(2) As shown in Fig. 1(b), a continuous sinusoidal signal of the normal force generated by the UMAC controller is used to move the $z$ axis stage up and down to ensure the constant depth of each cavity in the present study. The depth of each cavity is determined by the amplitude $(A)$ of the sinusoidal signal. Under this condition, the indentation process with a constant indentation depth is repeated.

(3) For procedure (2), if the $x-y$ stage does not move the sample, the tip indents the same location repeatedly. If the $x$ stage moves the sample with a velocity of $v_{x}$ simultaneously, as shown in Fig. 1(b), the continuous indentation processes will be separated. The distance between adjacent cavities is controlled by the period $(f)$ of the sinusoidal signal and the moving velocity of the $x$ stage $\left(v_{x}\right)$. Linear periodic micro/nano-cavities are generated with high efficiency using this method.

(4) The $y$ stage controls the sample to move to the other position after one line structure is fabricated, as shown in Fig. 1(c). Then, cavity arrays can be obtained, as shown in Fig. 1(b). The length and width of the microstructure array are controlled by the moving scales of the $x$ and $y$ stage, respectively.

\subsection{Measurement devices used in this study}

To perform Raman measurements, the structured copper samples were dipped into an R6G aqueous solution with a concentration of $10^{-6} \mathrm{M}$ for $30 \mathrm{~min}$ and then rinsed with ethanol to remove the excessive R6G molecules and dried with a continuous gentle nitrogen flow before Raman spectroscopy measurement. A micro-Raman spectroscopic system (Renishaw, inVia, UK) equipped with a $633 \mathrm{~nm}$ wavelength laser and focused with a $50 \times$ objective was employed. Approximately 6.4 $\mathrm{mW}$ laser power was used to excite the samples and the beam diameter was approximately $1 \mu \mathrm{m}$. The signal detector used a Renishaw CCD camera $(1040 \times 256)$ and the grating size was $1800 \mathrm{l} \mathrm{mm}^{-1}$. The exposure time was $1 \mathrm{~s}$ and the accumulation number was 1 . The mapping images of the micro-Raman spectrum were scanned over a $20 \mu \mathrm{m} \times 20 \mu \mathrm{m}$ area and the Raman mapping step size was controlled at $1 \mu \mathrm{m}$. Before the tests, a standard Si substrate was employed to rectify the Raman spectrum and no specific peaks were found. The Raman intensity of R6G was chosen at $1362 \mathrm{~cm}^{-1}$ in the experiment, which is the major peak intensity of R6G molecules.

A Dimension Icon AFM system (Bruker, Germany) was used to observe the topographies of the machined microstructures with the scan size of $50 \mu \mathrm{m} \times 50 \mu \mathrm{m}$. The elastic constant of the silicon cantilever was $0.2 \mathrm{~N} \mathrm{~m}^{-1}$ and contact mode was used.

\section{Results and discussion}

\subsection{Fabrication of arrayed cavities on the $\mathrm{Cu}(110)$ sample surface}

(1) The periodical force signal applied on the tip. Fig. 2 shows the force signals of the actual force, which is indicated by the blue line and the command force indicated by the pink line, 
(a)

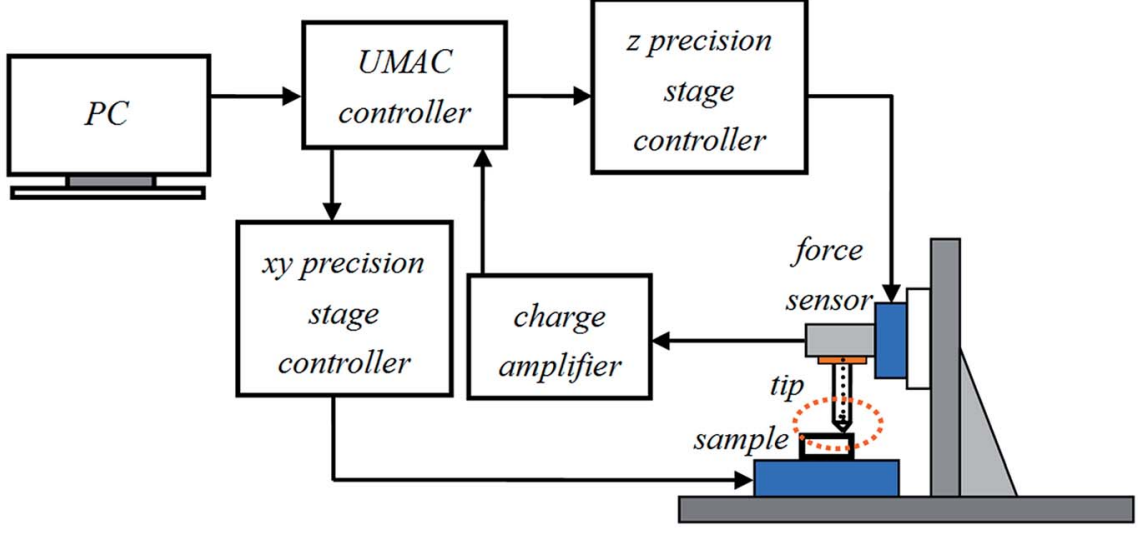

(b)

$z$ movement trajectory sine signal

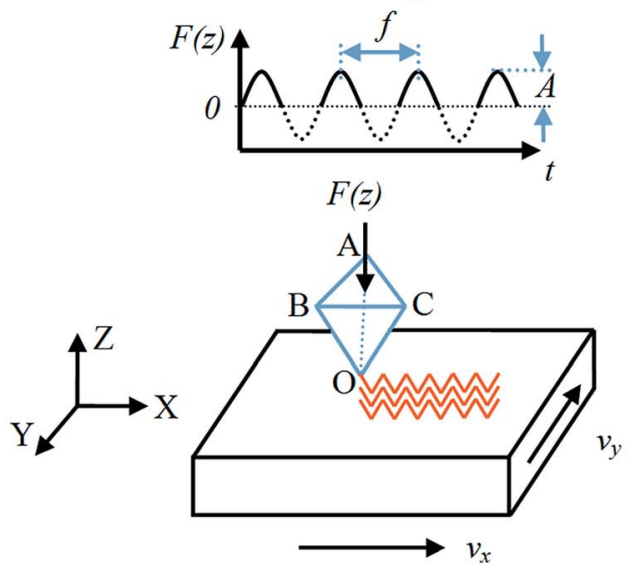

(c) $x$ y movement trajectories

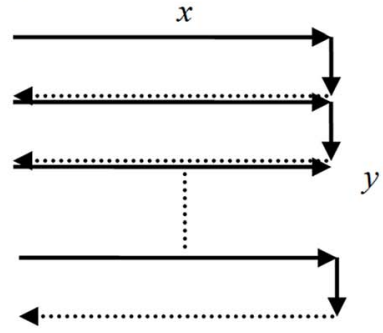

(d)

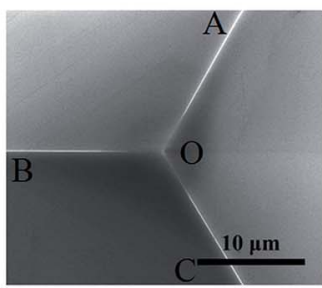

Fig. 1 (a) Schematic of the home-built tip-based force modulated indentation system. (b) Machining process for the fabrication of substrates with periodic micro/nano-cavities. (c) Movement trajectories of the $x$ and $y$ directions during machining. (d) SEM image of the cube corner tip with its shape.

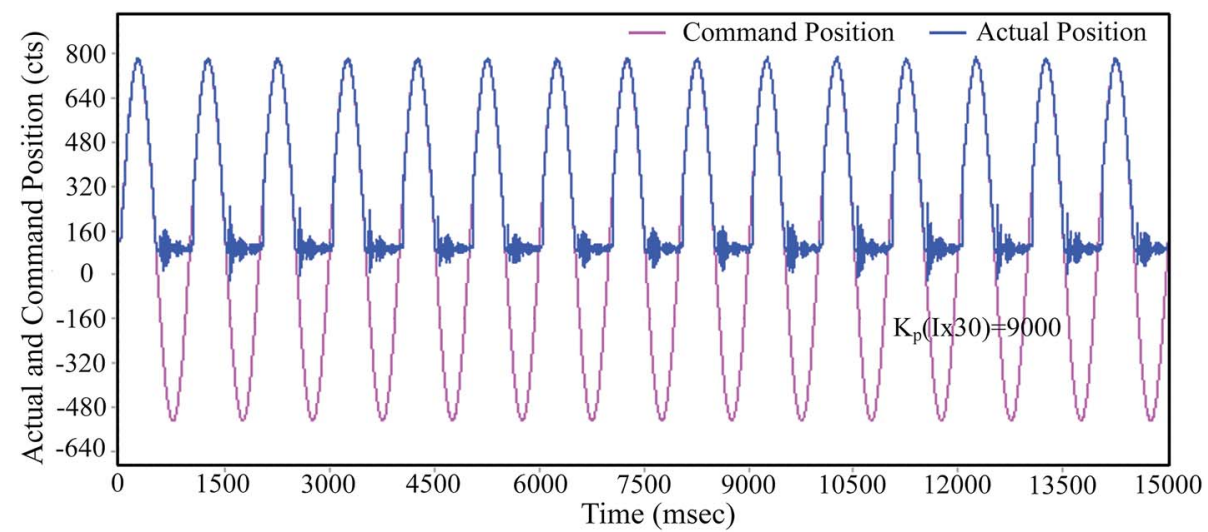

Fig. 2 Force signals of the actual force (blue line) and the command force (pink line).

applied on the tip during the machining process. This command force signal controls the $z$ axis nano accuracy stage to move through the UMAC controller. The amplitude $(A)$ and period $(f)$ of the force signal are $10 \mathrm{mN}$ and $1 \mathrm{~Hz}$, respectively. In the present study, both values are fixed, and only the machining velocity of the precision stage is varied to change the machined structures, which will be studied in detail in the following section. Moreover, as the blue line shows in Fig. 2, only a half 
signal is employed in the machining process. The amplitude of the other half sine signal is zero since the tip is pulled out from the sample surface at that time.

The depth is an important parameter of the micro cavity. The relationship between the normal force and the machined depth must be known. In the present study, the depth of the micro cavity is predicted by the hardness formula, which is used in previous studies. ${ }^{\mathbf{4 6 7}}$ The Berkovich hardness $(H)$ can be expressed as a function of the applied normal force $\left(F_{\mathrm{n}}\right)$ and the projected area $(A)$ of the cube corner tip after indentation, which is related to the penetration depth $(H)$ in eqn (1). The face angle with respect to the central axis of the diamond tip $(\alpha)$, the penetration depth $(h)$ and the projected area $(A)$ are expressed as eqn (2), where, $\alpha$ is $35.26^{\circ}$ for the cube corner tip, and the aluminum alloy hardness is $1.22 \mathrm{GPa}$, as measured using a Berkovich indenter. Therefore, the relationship between the normal force and the groove depth can be obtained from eqn (3) when using a cube corner tip. The groove depth is $1.79 \mu \mathrm{m}$ with the normal force of $10 \mathrm{mN}$.

$$
\begin{gathered}
H=\frac{F_{\mathrm{n}}}{A} \\
A=3 \sqrt{3} h^{2} \tan ^{2} \alpha
\end{gathered}
$$

The penetration depth, $h$, can then be obtained from eqn (3) using eqn (1) and (2):

$$
h=\sqrt{\frac{F_{\mathrm{n}}}{3 \sqrt{3} H \tan ^{2} \alpha}}
$$

(2) Effects of the moving velocity of the stage on the formation of the inverted pyramid cavities. Fig. 3 shows the AFM images of the inverted pyramid cavity (Fig. 3(a) and (c)) and the schematic shapes of the cavity (Fig. 3(b) and (d)) when the precision stage is fixed or moved with a velocity, respectively. When the stage is fixed at one position, the shape of the cavity agrees well with the triangular shape of the tip, as shown in Fig. 3(a) and (b). When the stage moves during the indentation process, the original triangle shape (the red triangle abc) transforms into a new one (the triangle abd indicated by the black dotted line) as shown in Fig. 3(d). The side length of the cavity changes from $5.12 \mu \mathrm{m}$ to $10.47 \mu \mathrm{m}$. Fig. 4 and 5 show the AFM images of single line arrayed inverted pyramid cavities machined with different moving velocities with the plane AOC or $\mathrm{AOB}$ as the rake face, respectively and Fig. 6 shows the relationship between the dimensions of cavities and the moving velocity along different machining directions.

(3) Fabrication of two dimensional inverted pyramid cavities arrays. Fig. 7 shows the AFM images of the arrayed inverted pyramid cavities with different machining velocities in the $x$ and $y$ directions. A schematic of the motion is presented in Fig. 1(c). The $y$ axis is used to obtain two dimensional microstructures. The normal force is the same as that mentioned in the previous section. The method of fabricating periodic arrayed cavities is different from the traditional indentation

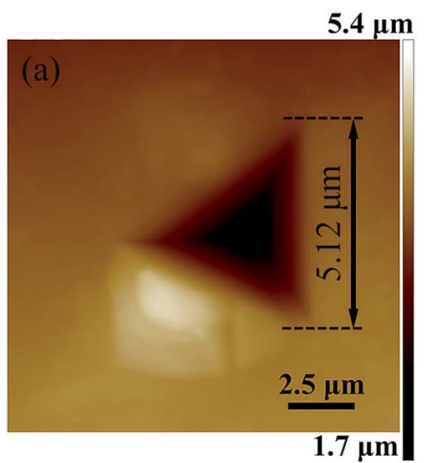

$5.4 \mu \mathrm{m}$

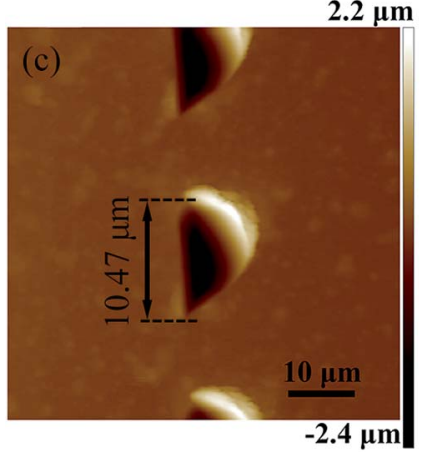

(d)

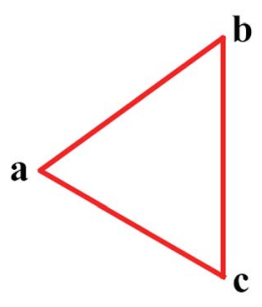

Fig. 3 (a) AFM images of the inverted pyramid cavity when the precision stage is fixed. (b) Schematic shapes of the cavity when the precision stage is fixed. (c) AFM images of the inverted pyramid cavity when the precision stage is moved. (d) Schematic shapes of the cavity when the precision stage is moved.

process. ${ }^{\mathbf{4 2 - 4 4}}$ Triangular cavities were fabricated on a silicon wafer using a traditional nanoindentor. However, complex structures of overlapped adjacent cavities have not been machined by researchers. ${ }^{42}$ Some researchers ${ }^{43,44}$ machined similar inverted triangular Au cavity arrays and structures with different overlapped cavities were obtained by controlling the distance of adjacent cavities. The $z$ and $x$ precision stages do not move simultaneously during machining using a traditional nanoindentor. However, our setup can work such that the $z$ and $x$ precision stages simultaneously move to fabricate cavities with high machining efficiency. The velocity $\left(v_{x}\right)$ of the $x$ stage ranges from $2 \mu \mathrm{m} \mathrm{s}^{-1}$ to $10 \mu \mathrm{m} \mathrm{s}^{-1}$ and the velocity $\left(v_{y}\right)$ of the $y$ stage ranges from $1 \mu \mathrm{m} \mathrm{s}^{-1}$ to $10 \mu \mathrm{m} \mathrm{s}^{-1}$. The areas of the periodic arrayed cavities are $30 \times 30 \mu \mathrm{m}^{2}$. The adjacent pyramid inverted cavities are separated using higher velocities, as shown in Fig. $7(\mathrm{a})$. When both velocities $\left(v_{x}\right.$ and $\left.v_{y}\right)$ are decreased to $5 \mu \mathrm{m} \mathrm{s}^{-1}$, the pile-ups of the adjacent inverted cavities induced by the machining are overlapped, as shown in Fig. 7(b). More complex nanostructures are formed, as shown in Fig. 7(c)-(f), because the adjacent cavities are overlapped and squeezed using this method with a decrease in velocity $\left(v_{y}\right)$. The projected surface area of the inverted triangular micro-cavity gradually becomes smaller in the $y$ direction with a decrease in velocity $\left(v_{y}\right)$, as shown in Fig. 7(c)-(f). In addition, the inverted triangular cavities change into two parts and form a structure similar to a "fish scale" in the range of velocity $\left(v_{y}\right)$ between 1 and $2 \mu \mathrm{m} \mathrm{s}^{-1}$, as shown in Fig. 7(e) and (f). During this process, the velocity $\left(v_{x}\right)$ is fixed at $5 \mu \mathrm{m} \mathrm{s}^{-1}$. It is the same 

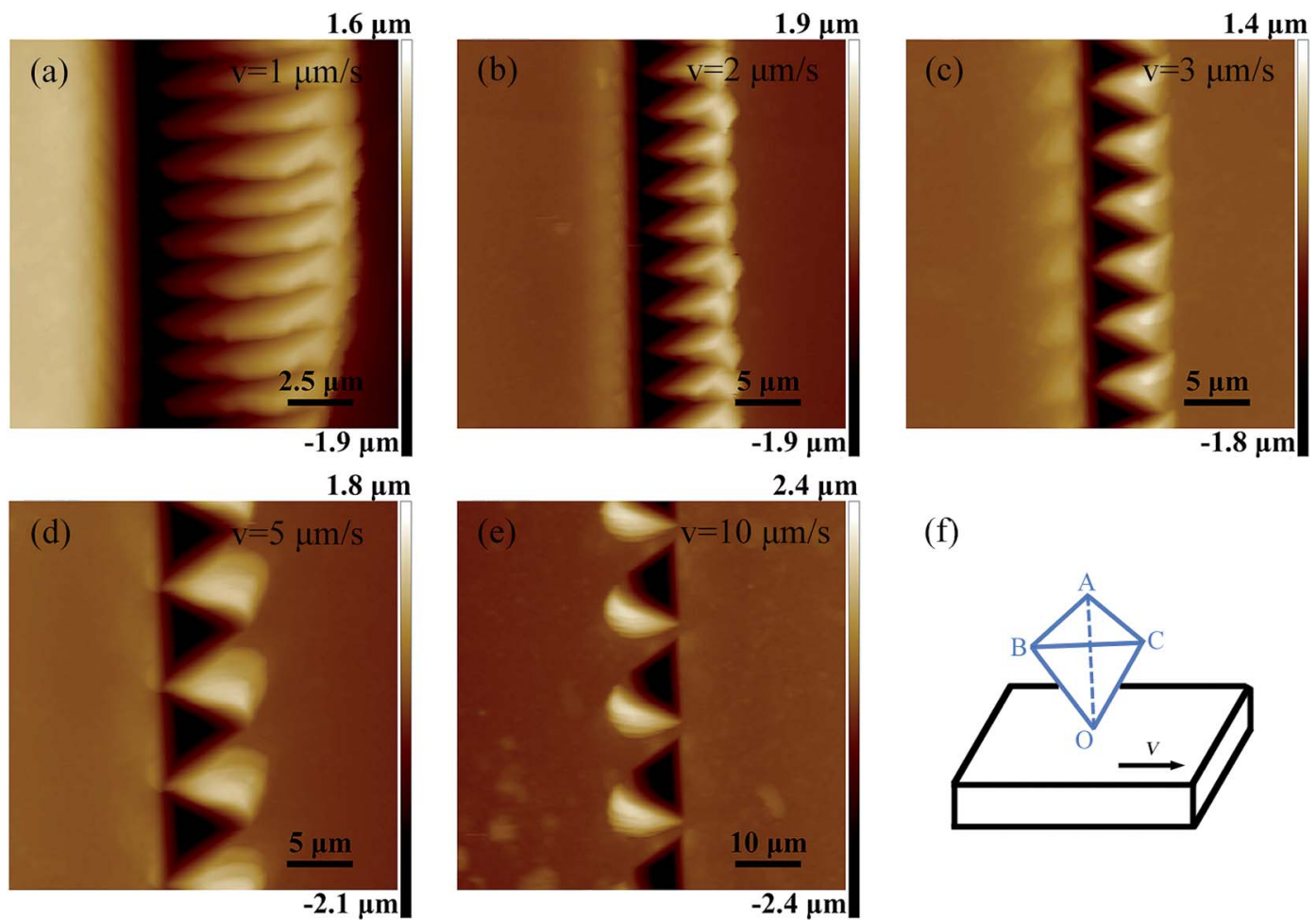

(f)

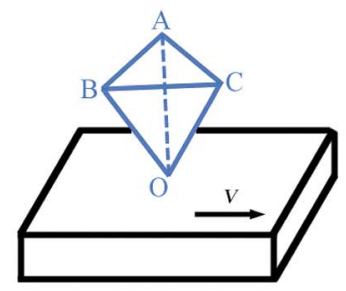

Fig. 4 AFM images of the arrayed inverted pyramid cavities with different machining velocities with the plane AOC of the cube corner tip as the rake face (a)-(e) the machining velocity of 2, 3, 4, 5 and $10 \mu \mathrm{m} \mathrm{s}^{-1}$, respectively. (f) Schematic machining direction with the plane $A O C$ of the cube corner tip.

$1.1 \mu \mathrm{m}$
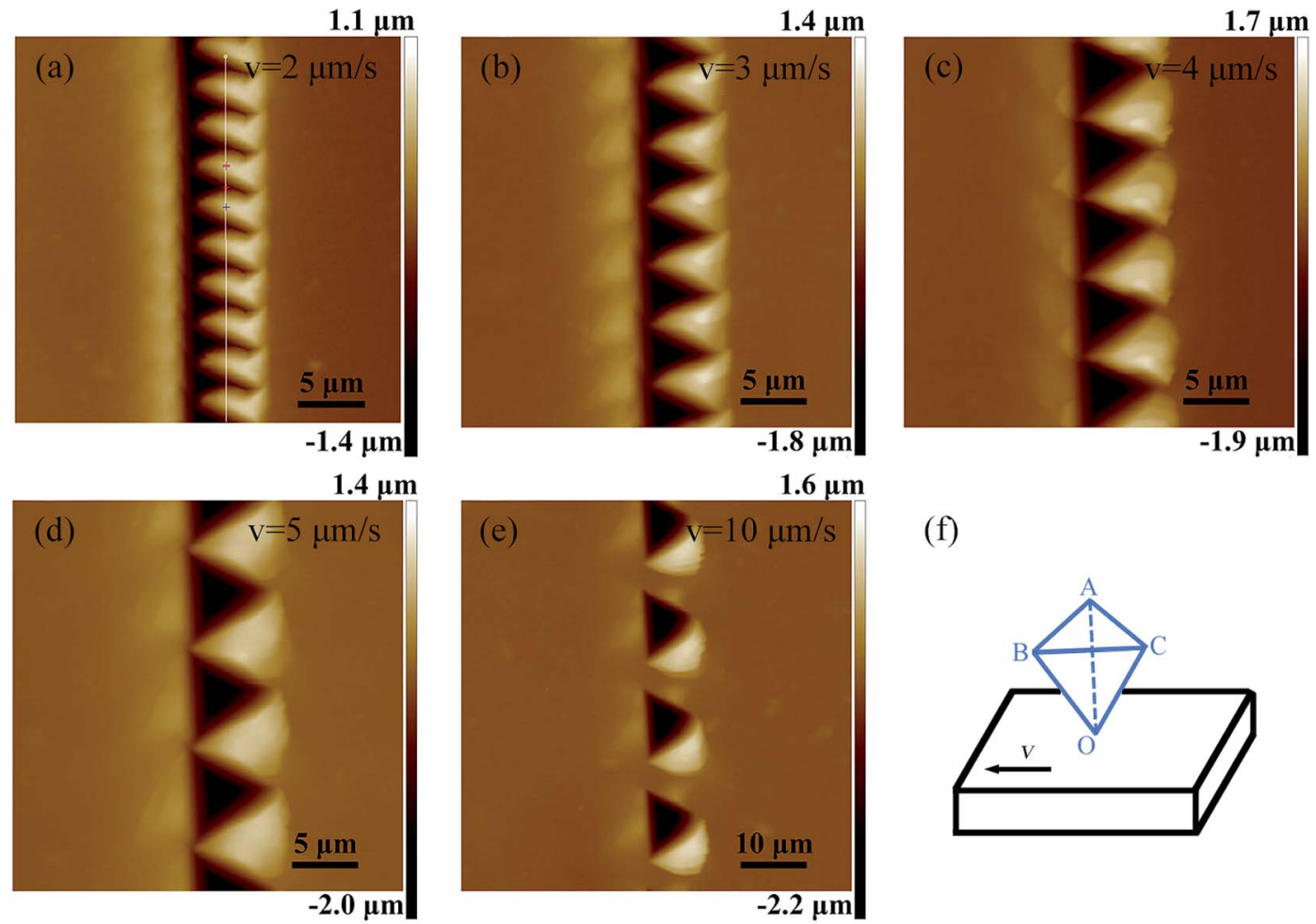

(f)

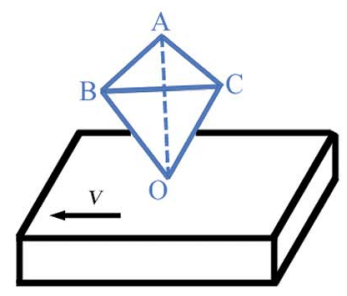

Fig. 5 AFM images of arrayed inverted pyramid cavities with different machining velocities with the plane AOB of the cube corner tip as the rake face (a)-(e) the machining velocity of $2,3,4,5$ and $10 \mu \mathrm{m} \mathrm{s}^{-1}$, respectively. (f) Schematic machining direction with the plane AOC of the cube corner tip. 
(a)

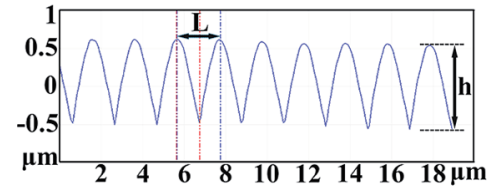

(b)

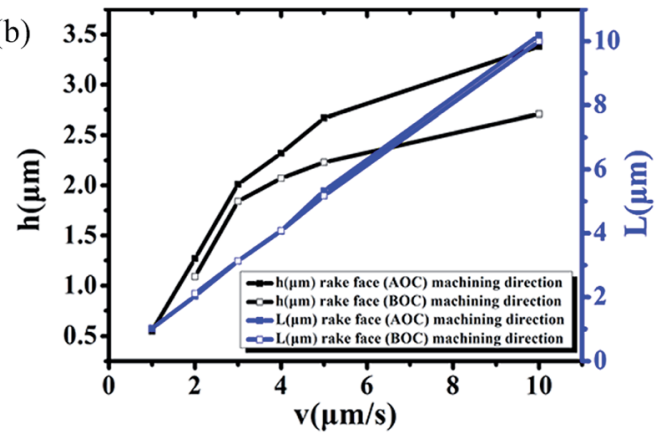

Fig. 6 (a) Schematic diagram of the depth and the width of cavities. (b) Relationship between the dimensions of the cavities and the moving velocity of the precision stage.
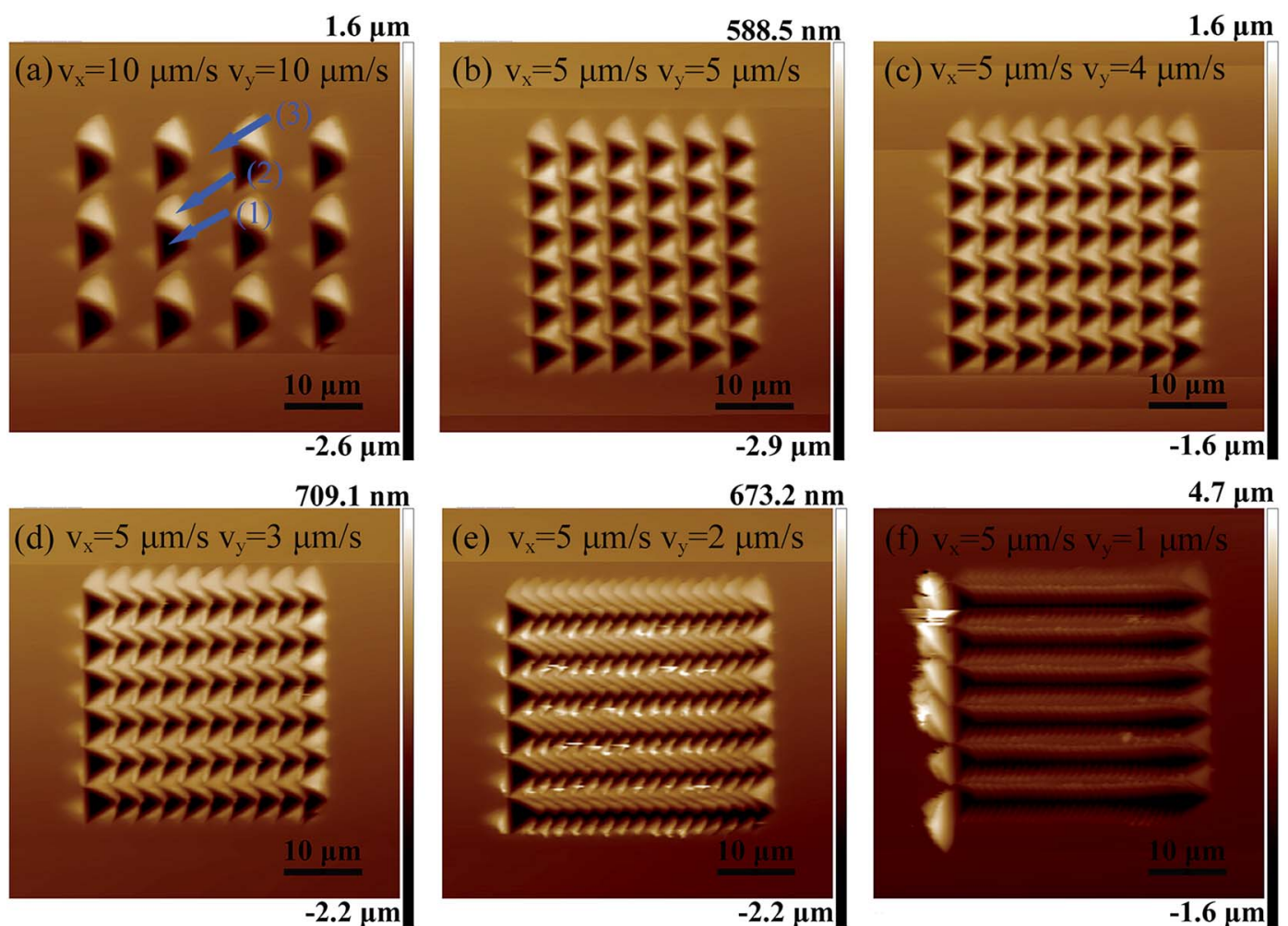

$673.2 \mathrm{~nm}$
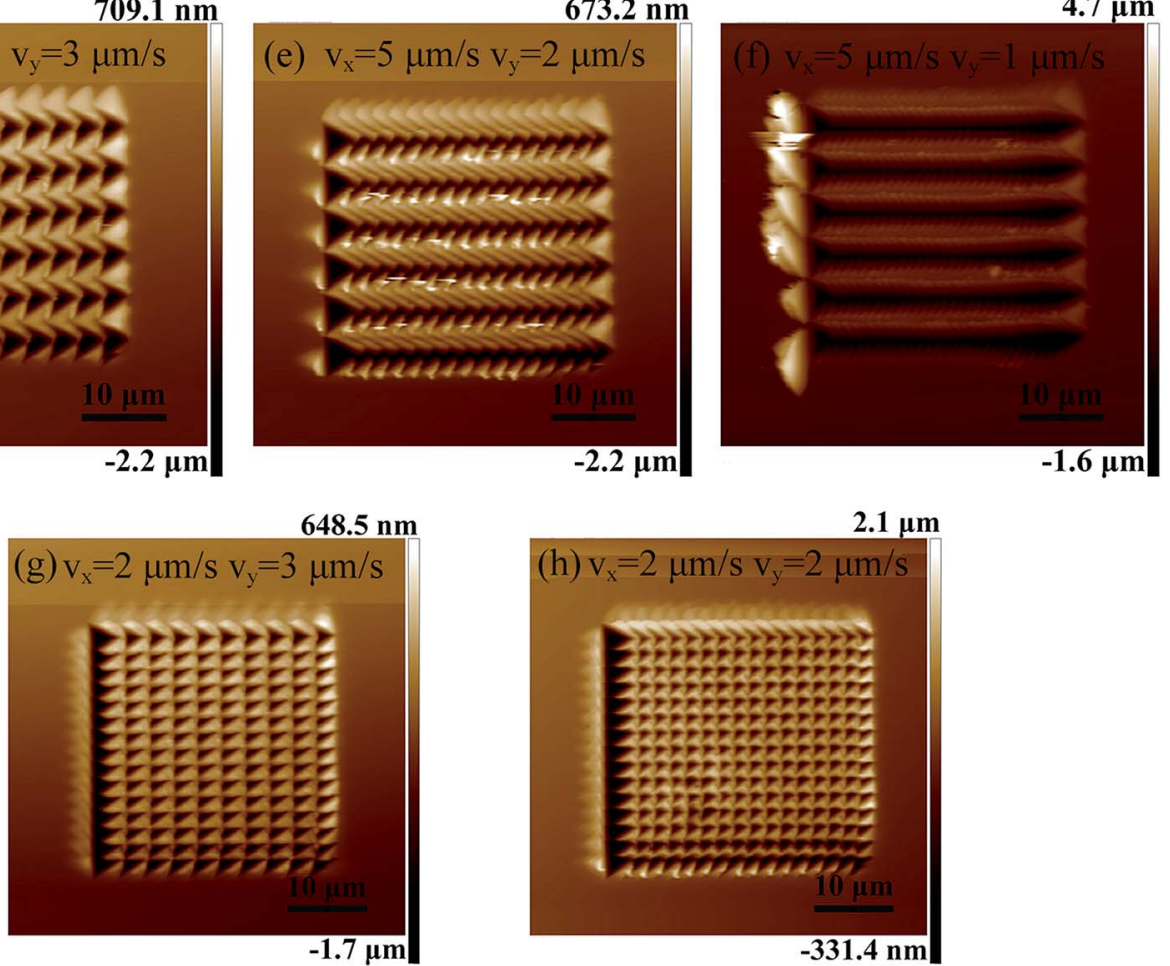

Fig. 7 AFM images of the arrayed inverted pyramid cavities with different velocities $\left(v_{x}\right.$ and $\left.v_{y}\right)$.

condition when the velocity $\left(v_{x}\right)$ varies. When the velocity $\left(v_{x}\right)$ ranges from 5 to $2 \mu \mathrm{m} \mathrm{s}^{-1}$, the structures of the inverted triangular cavities change and form smaller triangular cavities with a wide range, as shown in Fig. $7(\mathrm{~g})$ and $(\mathrm{h})$. The structures formed by this method have good reproducibility and homogeneity. 
Table 1 Parameters of the velocities of the $x-y$ stage for machining the arrayed micro/nano structures with a fixed normal force of $10 \mathrm{mN}$ and 1 $\mathrm{Hz}$

\begin{tabular}{lllllllllllllll}
\hline & $(1)$ & $(2)$ & $(3)$ & $(4)$ & $(5)$ & $(6)$ & $(7)$ & $(8)$ & $(9)$ & $(10)$ & $(11)$ & $(12)$ & $(13)$ & $(14)$ \\
\hline$v_{x}\left(\mu \mathrm{m} \mathrm{s}^{-1}\right)$ & 10 & 5 & 5 & 5 & 5 & 5 & 4 & 4 & 4 & 4 & 2 & 2 & 2 \\
$v_{y}\left(\mu \mathrm{m} \mathrm{s}^{-1}\right)$ & 10 & 5 & 4 & 3 & 2 & 1 & 4 & 3 & 2 & 1 & 4 & 3 & 2 \\
\hline
\end{tabular}
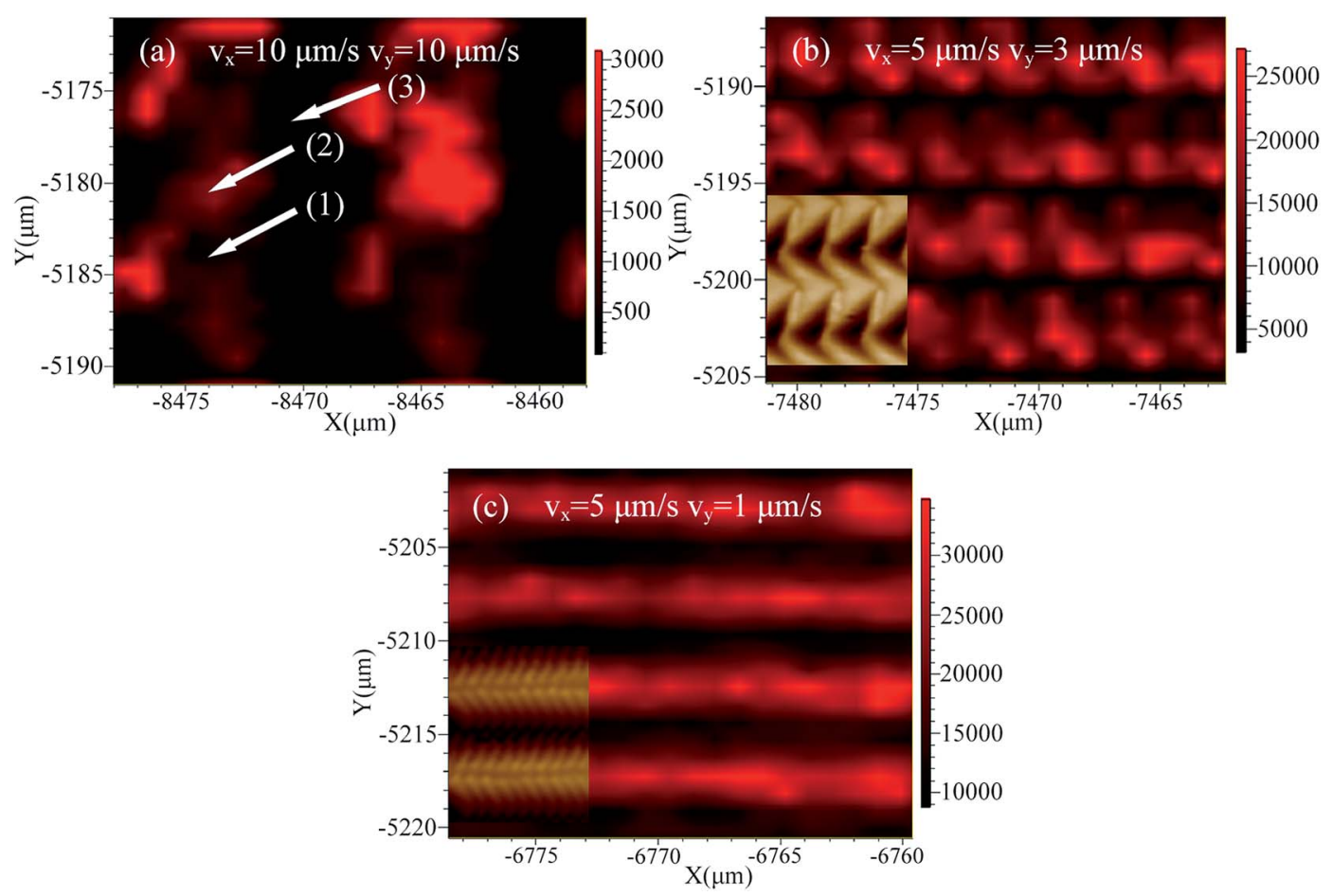

Fig. 8 Raman intensity mapping images of the arrayed inverted pyramid cavities fabricated with three moving velocities of the stage (a) velocity of $10 \mu \mathrm{m} \mathrm{s}^{-1}$ in both $x$ and $y$ directions. (b) Velocity of $5 \mu \mathrm{m} \mathrm{s}^{-1}$ in the $x$ direction and $3 \mu \mathrm{m} \mathrm{s}^{-1}$ in the $y$ direction and the inset is the AFM image corresponding to the field map. (c) Velocity of $5 \mu \mathrm{m} \mathrm{s}^{-1}$ in the $x$ direction and $1 \mu \mathrm{m} \mathrm{s}^{-1}$ in the $y$ direction and the inset is an AFM image of the structure of the "fish scale" corresponding to the field map.

\subsection{Raman enhancement of R6G molecules on the arrayed inverted pyramid cavities on $\mathrm{Cu}(110)$ substrates}

In order to study the effect of Raman enhancement of R6G molecules with different arrayed inverted pyramid cavities, the parameters of the velocities of the $x-y$ stage for machining arrayed micro/nano structures with a fixed normal force of 10 $\mathrm{mN}$ and $1 \mathrm{~Hz}$ using the method presented in this study are shown in Table 1. Fig. 8 shows the Raman intensity mapping images of absorbed R6G (1362 $\mathrm{cm}^{-1}$ peak) on the arrayed inverted pyramid cavities with three moving velocities of the stage in the $x$ and $y$ directions. The velocity $\left(v_{x}\right)$ ranges from 5 $\mu \mathrm{m} \mathrm{s}^{-1}$ to $10 \mu \mathrm{m} \mathrm{s}^{-1}$ and the velocity $\left(v_{y}\right)$ ranges from $1 \mu \mathrm{m} \mathrm{s}^{-1}$ to $10 \mu \mathrm{m} \mathrm{s}^{-1}$. Raman spectra were collected with a step size of 1 $\mu \mathrm{m}$ along the $x$ and $y$ directions by a point mapping pattern. At each point, the spectra were collected for $1 \mathrm{~s}$ over two scans. The brighter the pixel, the higher the intensity. SERS spectra are observed in both the interior of the cavities and pile-ups formed near the cavities, as indicated by arrows (1) and (2) in Fig. 8(a), which correspond to the positions of arrows (1) and (2) in
Fig. 7(a). However, there is no significant enhancement in the $\mathrm{Cu}$ (110) surface, as shown by arrow (3) in Fig. 8(a), which corresponds to the position of arrow (3) in Fig. 7(a). Essentially, the flat $\mathrm{Cu}$ surface, rather than the overlapped cavities, remained in this area with a larger moving velocity. The insets in Fig. 8(b) and (c) are AFM images corresponding to the field map. The correspondence between the topography of the "fish scale" profile and the near-field distribution mapping are clearly seen in Fig. 8(c). It is experimentally verified that the distributions of the Raman intensities are uniform along the same machining direction and it is demonstrated that this novel tip-based method has high reproducibility and reliability, as shown in Fig. 8(b) and (c).

The data of Raman mapping were exported to Raman spectra point by point. Prior to analysis, first, all the Raman spectra were smoothened using a Savitzky-Golay filter with a thirdorder polynomial and a smooth window size of 13. Second, the baseline of the Raman spectra was removed by subtracting a spline interpolation using WiRE 3.4. 
(a)

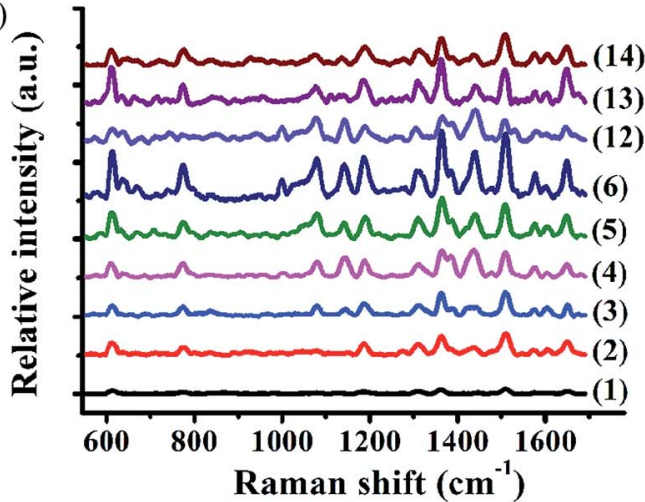

(b)

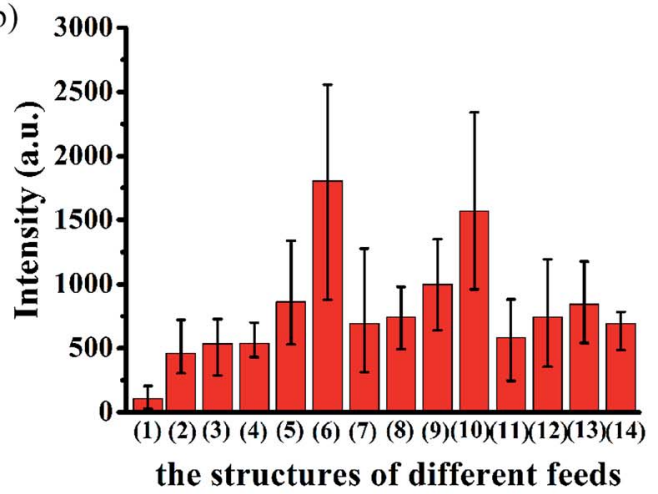

Fig. 9 (a) Raman spectra of R6G molecules on the structured $\mathrm{Cu}$ (110) substrate at a concentration of $10^{-6} \mathrm{M}$ with different machining velocities. (b) Average Raman intensity of the $1362 \mathrm{~cm}^{-1}$ R6G peak of $20 \mu \mathrm{m} \times 20 \mu \mathrm{m}$ of arrayed inverted pyramid cavities with different machining velocities.

Fig. 9 shows the Raman spectra of R6G molecules on the structured $\mathrm{Cu}$ (110) substrate at a concentration of $10^{-6} \mathrm{M}$ under different machining conditions. The characteristic peaks of the R6G molecules are shown at $612 \mathrm{~cm}^{-1}, 771 \mathrm{~cm}^{-1}, 1183$ $\mathrm{cm}^{-1}, 1311 \mathrm{~cm}^{-1}, 1362 \mathrm{~cm}^{-1}, 1504 \mathrm{~cm}^{-1}$ and $1603 \mathrm{~cm}^{-1}$. It can be observed that the Raman enhancement can be remarkably affected by the machining velocities in the $x$ and $y$ directions when the applied force has a constant variation rate.

All the characteristic peaks of the Raman intensity in curve (1) have a weaker enhancement in Fig. 9(a). This agrees well with the results shown in Fig. 8(a). Under this condition, the distance between the adjacent cavities is too large for them to be overlapped with each other, as shown in Fig. 7(a). The Raman intensity with the peak of $1362 \mathrm{~cm}^{-1}$ R6G increases with a decrease in velocity in the $y$ direction, as shown by the curves (2)-(6) in Fig. 9(a). The Raman intensity with the velocity $\left(v_{y}\right)$ of 1 $\mu \mathrm{m} \mathrm{s}^{-1}$ is the strongest.

Fig. 10(b)-(d) show an enlarged AFM image of the arrayed inverted pyramid cavities and three-dimensional and section views of the structures with the velocity $\left(v_{x}\right)$ of $5 \mu \mathrm{m} \mathrm{s}^{-1}$ and velocity $\left(v_{y}\right)$ of $1 \mu \mathrm{m} \mathrm{s}^{-1}$, respectively. The depths of the nanostructures are $116 \mathrm{~nm}, 233 \mathrm{~nm}$ and $260 \mathrm{~nm}$ in Fig. 10(d). In addition, the microstructures induced by the tip's trace are homogeneously distributed on the $\mathrm{Cu}(110)$ surface. The Raman intensity with the peak of $1362 \mathrm{~cm}^{-1} \mathrm{R} 6 \mathrm{G}$ does not significantly change and is weaker than that of the structures with the velocity $\left(v_{x}\right)$ of $5 \mu \mathrm{m} \mathrm{s}^{-1}$ and velocity $\left(v_{y}\right)$ of $1 \mu \mathrm{m} \mathrm{s}^{-1}$, as shown by the curves (12)-(14) in Fig. 9(a). Fig. 9(b) shows the average
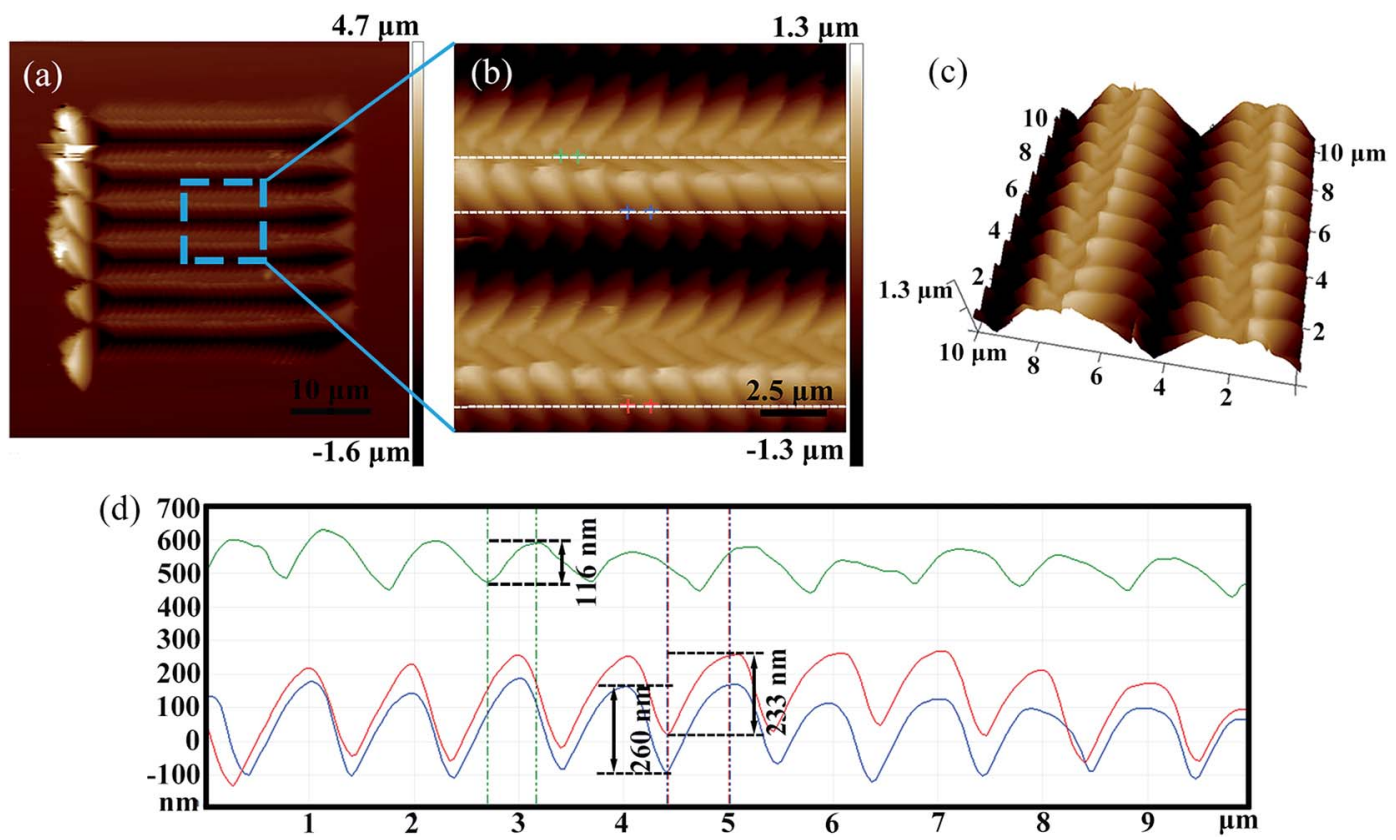

Fig. 10 (a) AFM images of arrayed inverted pyramid cavities machined with $v_{x}=5 \mu \mathrm{m} \mathrm{s} \mathrm{s}^{-1}$ and $v_{y}=1 \mu \mathrm{m} \mathrm{s} \mathrm{s}^{-1}$. (b) Enlarged image of the local position of figure (a). (c) Three-dimensional view of structures with $v_{x}=5 \mu \mathrm{m} \mathrm{s}^{-1}$ and $v_{y}=1 \mu \mathrm{m} \mathrm{s}{ }^{-1}$. (d) Sectional view of the structures in figure (b). 
Raman intensity of the $1362 \mathrm{~cm}^{-1}$ R6G of $20 \mu \mathrm{m} \times 20 \mu \mathrm{m}$ of the arrayed inverted pyramid cavities with different machining velocities, which correspond to Fig. 9(a). The structures of inverted cavities change on using different machining velocities. In addition, nanostructures obtained using different machining velocities are formed by the overlap and extrusion of the adjacent pyramid cavities. The depth of the nanostructures obtained using a velocity $\left(v_{x}\right)$ of $5 \mu \mathrm{m} \mathrm{s}^{-1}$ and velocity $\left(v_{y}\right)$ of $1 \mu \mathrm{m} \mathrm{s}^{-1}$ is shallower than the depths of the nanostructures obtained using other machining parameters.

Therefore, the average intensity of the velocity $\left(v_{x}\right)$ of $5 \mu \mathrm{m}$ $\mathrm{s}^{-1}$ and velocity $\left(v_{y}\right)$ of $1 \mu \mathrm{m} \mathrm{s}^{-1}$ with the "fish scale" structure is the strongest, as shown in Fig. 9(b). When the velocity changes from $5 \mu \mathrm{m} \mathrm{s}^{-1}$ to $3 \mu \mathrm{m} \mathrm{s}^{-1}$, the intensity does not significantly change in Fig. 9(b). Previous studies obtained similar results. On one hand, more nanostructures are formed by different machining velocities, in particular the velocity of $5 \mu \mathrm{m} \mathrm{s}^{-1}$ in the $x$ direction and $1 \mu \mathrm{m} \mathrm{s}^{-1}$ in the $y$ direction, as shown in Fig. 7(f) and 10. Chang et al. ${ }^{\mathbf{4}}$ showed that probe molecules are easily entrapped into the nanostructures and noticeably detected by the SERS effect. Therefore, it can be deduced that more R6G molecules can be adsorbed and entrapped by the nanostructures. On the other hand, with proper excitation of this absorption, a strong local electromagnetic field with the electromagnetic mechanism (EM) is generated with different structures. Compared with the bare surface, an electric field is generated and a plasmon may exist on the interior of the single V-groove, as shown by some researchers, ${ }^{4-50}$ forming very strong near fields and enhancing the Raman signal, which corresponds to Fig. 7(a). Furthermore, a much stronger Raman enhancement apparently occurs in the vicinity of the nanostructures, as shown in Fig. 7(b)-(h). Some researchers ${ }^{51,52}$ have demonstrated that the electric field intensity is influenced by the distance between adjacent pyramids. The "hot lines" formed by two adjacent nanostructures are formed by an infinite number of hot spots. Compared with the dispersed structures, the electric field intensity generated by adjacent structures is about 15 times that of the electric field intensity generated by dispersed structures. ${ }^{51}$ Therefore, compared with dispersed structures, the uniformity and stability of the electric field intensity from the molecules adsorbed on the numerous

\section{Acknowledgements}

The authors gratefully acknowledge the financial supports of the Foundation for the National Natural Science Foundation of China (51675134), Innovative Research Groups of the National Natural Science Foundation of China (51521003), Self-Planned Task (SKLRS201606B) of State Key Laboratory of Robotics and System (HIT) and the National Program for Support of Topnotch Young Professors.

\section{References}

1 Z. L. Cao, S. L. Wong, S. Y. Wu, H. P. Ho and H. C. Ong, Appl. Phys. Lett., 2014, 104, 171116.

2 P. P. Huang, Z. Z. Wang, L. Lin, Z. H. Feng, X. Wen and Z. Q. Zheng, Appl. Surf. Sci., 2016, 386, 345-351.
Fig. 11 Raman spectra of $\mathrm{R} 6 \mathrm{G}$ molecules on the $\mathrm{SiO}_{2}$ substrate at a concentration of $0.25 \mathrm{M}$. hot lines formed by the nanostructures are significantly nhanced, which agrees well with our experiment results.

$$
\mathrm{EF}=\frac{I_{\mathrm{SERS}} \times N_{\mathrm{NR}}}{N_{\mathrm{SERS}} \times I_{\mathrm{NR}}}=\frac{I_{\mathrm{SERS}} \times C_{\mathrm{NR}}}{C_{\mathrm{SERS}} \times I_{\mathrm{NR}}}
$$

where $I_{\mathrm{SERS}}$ and $I_{\mathrm{NR}}$ are the intensity of SERS and normal Raman scattering intensity, respectively, and $N_{\mathrm{SERS}}$ and $N_{\mathrm{NR}}$ are the concentrations of SERS and normal Raman measurement, maximum $I_{\text {SERS }}$ and minimum $I_{\text {SERS }}$ are 2700 and 105, respecthe present study are between $10^{5}$ and $3 \times 10^{6}$ for the structured

\section{Conclusions}

A novel method based on the tip-based force modulated nanoindentation process is employed to fabricate two and three plane. By optimizing the $x-y$ precision stage and $z$ direction precision stage, complex arrayed micro cavities are achieved by ities. Then, these arrayed micro stuctures structures for SERS substrates. The SERS intensity induced by these types of nanostructures machined using different enhancement factors are between $10^{5}$ and $3 \times 10^{6}$ for the structured single crystal copper (110) plane, which demonstrates that this method is reliable, replicable, homogeneous and inexpensive for machining large-area regular nanostructures. Therefore, this tip-based force modulated indentation method is expected to develop new opportunities for labelfree SERS detection in biosensing and bioanalytics, bacteria and virus identification and the detection of explosives and toxic pesticides in the future.

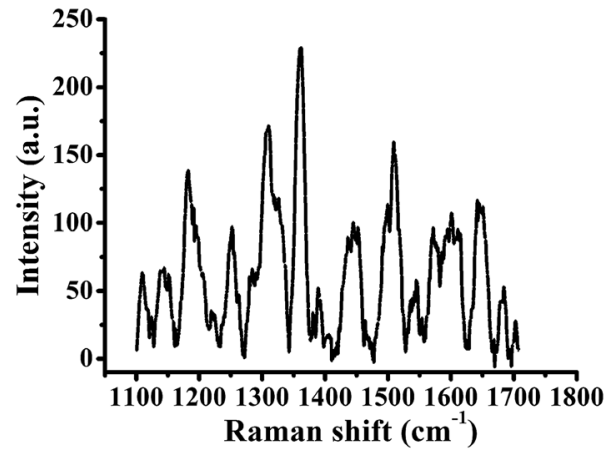


3 J. J. Zhang, J. G. Zhang, Z. F. Wang, A. Hartmaier, Y. D. Yan and T. Sun, Comput. Mater. Sci., 2017, DOI: 10.1016/ j.commatsci.2017.01.043.

4 Y. J. Oh and K. H. Jeong, Lab Chip, 2014, 14, 865-868.

5 A. Lee, G. H. Yeoh, V. Timchenko and J. A. Reizes, Appl. Therm. Eng., 2012, 48, 275-288.

6 J. Liu, X. S. Zhang, C. Q. Dong, Y. X. Liao, B. Wang, T. C. Zhang and F. T. Yi, Appl. Surf. Sci., 2014, 289, 300-305.

7 J. Liu, M. Ashmkhan, G. Q. Dong, B. Wang and F. T. Yi, Sol. Energy Mater. Sol. Cells, 2013, 108, 93-97.

8 S. Lombardo, C. Tringali, G. Cannella, A. Battaglia, M. Foti, N. Costa, F. Principato and C. Gerardi, Appl. Phys. Lett., 2012, 101, 123902.

9 C. A. Wolden, J. Kurtin, J. B. Baxter, I. Repins, S. E. Shaheen, J. T. Torvik, A. A. Rockett, V. M. Fthenakis and E. S. Aydil, J. Vac. Sci. Technol., A, 2011, 29, 030801.

10 W. Zhou, H. Gao and T. W. Odom, ACS Nano, 2010, 4, 12411247.

11 S. A. Kara, A. Keffous, A. M. Giovannozzi, A. M. Rossi, E. Cara, L. D'Ortenzi, K. Sparnacci, L. Boarino, N. Gabouze and S. Soukane, RSC Adv., 2016, 6, 93649-93659.

12 S. Z. Oo, S. Siitonen, V. Kontturi, D. A. Eustace and M. D. B. Charlton, Opt. Express, 2016, 24, 724-731.

13 J. Y. Lin, A. D. Stuparu, M. D. Huntington, M. Mrksich and T. W. Odom, J. Phys. Chem. C, 2013, 117, 5286-5292.

14 K. Nakamoto, R. Kurita, O. Niwa, T. Fujiicd and M. Nishida, Nanoscale, 2011, 3, 5067-5075.

15 T. A. Alexander, Anal. Chem., 2008, 80, 2817-2825.

16 K. Karn-orachai, K. Sakamoto, R. Laocharoensuk, S. Bamrungsap, S. Songsivilai, T. Dharakul and K. Miki, RSC Adv., 2016, 6, 97791-97799.

17 S. Botti, S. Almaviva, L. Cantarini, A. Palucci, A. Puiu and A. Rufoloni, J. Raman Spectrosc., 2013, 44, 463-468.

18 A. Hakonen, P. O. Andersson, M. S. Schmidt, T. Rindzevicius and M. Käll, Anal. Chim. Acta, 2015, 893, 1-13.

19 L. Wu, Z. Wang and B. Shen, Nanoscale, 2013, 5, 5274-5278.

20 M. L. Jin, V. Pully, C. Otto, A. V. D. Berg and E. T. Carlen, J. Phys. Chem. C, 2010, 114, 21953-21959.

21 A. Kinkhabwala, Z. F. Yu, S. H. Fan, Y. Avlasevich, K. Müllen and W. E. Moerner, Nat. Photonics, 2009, 3, 654-657.

22 J. Petschulat, D. Cialla, N. Janunts, C. Rockstuhl, U. Hübner, R. Möller, H. Schneidewind, R. Mattheis, J. Popp, A. Tünnermann, F. Lederer and T. Pertsch, Opt. Express, 2010, 18, 4184-4197.

23 J. H. Zhang, Y. F. Li, X. M. Zhang and B. Yang, Adv. Mater., 2010, 22, 4249-4269.

24 J. Y. Park, Korean J. Chem. Eng., 2014, 31, 541-547.

25 Z. Tang and A. Wei, ACS Nano, 2012, 6, 998-1003.

26 H. Gao, C. Liu, H. E. Jeong and P. D. Yang, ACS Nano, 2011, 6, 234-240.

27 K. Nakamoto, R. Kurita, O. Niwa, T. Fujii and M. Nishida, Nanoscale, 2011, 3, 5067-5075.

28 H. Gao, J. C. Yang, J. Y. Lin, A. D. Stuparu, M. H. Lee, M. Mrksich and T. W. Odom, Nano Lett., 2010, 10, 25492554.
29 T. W. Odom, MRS Bull., 2010, 35, 66-73.

30 W. Zhou, H. Gao and T. W. Odom, ACS Nano, 2010, 4, 12411247.

31 Z. D. Zhu, Q. Q. Li, B. F. Bai and S. S. Fan, Nanoscale Res. Lett., 2014, 9, 1-8.

32 T. J. Wang, K. C. Hsu, Y. C. Liu, C. H. Lai and H. P. Chiang, J. Opt., 2016, 18, 055006.

33 M. Jin, H. V. Wolferen, H. Wormeester, A. V. D. Berga and E. T. Carlen, Nanoscale, 2012, 4, 4712-4718.

34 Y. L. Ho, M. Abasaki and J. J. Delaunay, ACS Photonics, 2015, 2, 730-737.

35 S. S. Ye, X. M. Zhang, L. X. Chang, T. Q. Wang, Z. B. Li, J. H. Zhang and B. Yang, Adv. Opt. Mater., 2014, 2, 779-787.

36 H. Im, S. H. Lee, N. J. Wittenberg, T. W. Johnson, N. C. Lindquist, P. Nagpal, D. J. Norris and S. H. Oh, ACS Nano, 2011, 5, 6244-6253.

37 H. W. Gao, J. Henzie, M. H. Lee and T. W. Odom, Proc. Natl. Acad. Sci. U. S. A., 2008, 105, 20146-20151.

38 B. K. Chao, H. H. Cheng, L. W. Nien, M. J. Chen, T. Nagao, J. H. Li and C. H. Hsueh, Appl. Surf. Sci., 2015, 357, 615-621.

39 K. C. Vernon, T. J. Davis, F. H. Scholes, D. E. Gómez and D. Lau, J. Raman Spectrosc., 2010, 41, 1106-1111.

40 J. M. Li, X. X. Xu, Y. F. Wang, M. Wang, Z. F. Dong, W. H. Tian, J. M. Sun, C. Z. Zhang and B. Wang, J. Raman Spectrosc., 2012, 43, 863-868.

41 S. Botti, L. Cantarini, S. Almaviva, A. Puiub and A. Rufolonic, Chem. Phys. Lett., 2014, 592, 277-281.

42 J. L. Gong, D. J. Lipomi, J. D. Deng, Z. H. Nie, X. Chen, N. X. Randall, R. Nair and G. M. Whitesides, Nano Lett., 2010, 10, 2702-2708.

43 C. W. Chang, J. D. Liao, A. L. Shiau and C. K. Yao, Sens. Actuators, B, 2011, 156, 471-478.

44 C. K. Yao, J. D. Liao, C. W. Chang and J. R. Lin, Sens. Actuators, B, 2012, 174, 478-484.

45 C. K. Yao, J. D. Liao, C. H. Lin, Y. S. Yang, S. H. Yu and J. W. Yang, Sens. Actuators, B, 2014, 191, 219-226.

46 A. C. Fischer-Cripps and I. Mustafaev, Introduction to contact mechanics, Springer, New York, 2000.

47 J. M. Lee, W. H. Jin and D. E. Kim, Wear, 2001, 251, 11331143.

48 K. C. Vernon, T. J. Davis, F. H. Scholes, D. E. Gómez and D. Lau, J. Raman Spectrosc., 2010, 41, 1106-1111.

49 C. L. C Smith, B. Desiatov, I. Goykmann, I. FernandezCuesta, U. Levy and A. Kristensen, Opt. Express, 2012, 20, 5696-5706.

50 F. H. Scholes, T. J. Davis, K. C. Vernon, D. Lau, S. A. Furman and A. M. Glenn, J. Raman Spectrosc., 2012, 43, 196-201.

51 R. Li, H. Li, S. Pan, K. Liu, S. S. Hu, L. J. Pan, Y. N. Guo and S. F. Wu, J. Mater. Res., 2013, 28, 3401-3407.

52 H. H. Cheng, S. W. Chen, Y. Y. Chang, J. Y. Chu, D. Z. Lin, Y. P. Chen and J. H. Li, Opt. Express, 2011, 19, 22125-22141. 53 Q. Shao, R. H. Que, M. W. Shao, L. Cheng and S. T. Lee, Adv. Funct. Mater., 2012, 22, 2067-2070.

54 W. B. Cai, B. Ren, X. Q. Li, C. X. She, F. M. Liu, X. W. Cai and Z. Q. Tian, Surf. Sci., 1998, 406, 9-22. 